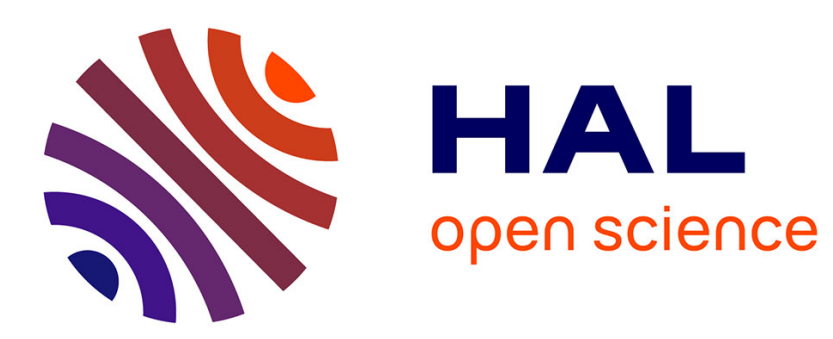

\title{
Flow streamline based Navier-Stokes Characteristic Boundary Conditions: Modeling for transverse and corner outflows
}

Eric Albin, Yves d'Angelo, Luc Vervisch

\section{- To cite this version:}

Eric Albin, Yves d'Angelo, Luc Vervisch. Flow streamline based Navier-Stokes Characteristic Boundary Conditions: Modeling for transverse and corner outflows. Computers and Fluids, 2011, 51 (1), pp.115-126. 10.1016/j.compfluid.2011.08.005 . hal-00967141

\section{HAL Id: hal-00967141 \\ https://hal.science/hal-00967141}

Submitted on 28 Mar 2014

HAL is a multi-disciplinary open access archive for the deposit and dissemination of scientific research documents, whether they are published or not. The documents may come from teaching and research institutions in France or abroad, or from public or private research centers.
L'archive ouverte pluridisciplinaire $\mathbf{H A L}$, est destinée au dépôt et à la diffusion de documents scientifiques de niveau recherche, publiés ou non, émanant des établissements d'enseignement et de recherche français ou étrangers, des laboratoires publics ou privés. 


\title{
Flow streamline based Navier-Stokes Characteristic Boundary Conditions: modeling for transverse and corner outflows
}

\author{
Eric Albin, Yves D’Angelo \& Luc Vervisch \\ INSA de Rouen, CORIA - CNRS 76801 St Etienne du Rouvray, Rouen, France
}

\begin{abstract}
Some limitations of three dimensional Navier-Stokes Characteristic Boundary Conditions (3DNSCBC) are discussed for flows traveling in a direction that is oblique to the boundary. To limit errors generated at boundaries with flows having any arbitrary direction, it is proposed to organize the wave decomposition in a coordinate system that is attached to the local flow streamline crossing the boundary, because some modeled expressions are not frame independent. Compared to previous 3D-NSCBC, the modified strategy accounting for oblique waves is found to improve the outflow treatment for transverse outgoing vortices, up to vortices crossing an outflow corner. The method is also applied to an expanding laminar flame.
\end{abstract}

Keywords: Boundary conditions, Direct Numerical Simulation, Large Eddy Simulation

\section{Introduction}

In 1987, Thompson [1] introduces multi-dimensional boundary treatment for Euler equations. He makes use of a characteristic formulation of hyperbolic conservation equations: outcoming waves are computed from their analytic expressions, while incoming waves amplitudes are prescribed zero values. Poinsot and Lele [2] extend this procedure to Navier-Stokes equations and introduce the so-called NSCBC treatment (for Navier-Stokes Characteristic Boundary Conditions). They assume the fluid to be locally inviscid, non reactive, and without transverse convective terms. They insist upon the number of boundary conditions needed to be set in order to get a well-posed problem. Yoo et al $[3,4]$ include transverse, diffusive and reactive terms in the modeling of ingoing waves. Recently, Lodato et al [5] apply this methodology to Large Eddy Simulation (LES) [6] and introduce a particular strategy for edges and corners, leading to a 3D-NSCBC modeling. However, the use of the Lodato et al. BC provides some improvements at edges and corner, but does not fully address the coordinate system issue discussed in this work. In 2009, Liu \& Vasilyev [7] state a different multi-dimensional characteristic formulation, that separates and will then treat differently characteristic wave convective terms from others, considered as "source terms". Due to acoustic incoming — i.e. from the outside — waves, these "source terms" are specifically damped with the help of adjusted coefficients for acoustic transverse waves not to be reflected. As in the present work, these authors suggest that observed spurious reflections of acoustic waves at outflows are mainly caused by inappropriate treatment of compressible boundaries, especially in the presence of multidimensional non-linearities. Many other developments have been devoted to boundary conditions for more or less compressible flows $[8,9,10,11]$. 
The characteristic form of the Euler equations, serving as a basis to develop compressible boundary conditions, is coordinate system independent; however, isolated terms (corresponding to a given component) cannot be frame invariant. Their isolated modeled expression therefore depends on the chosen coordinate system and it is shown that the choice of the flow streamline as coordinate system is an interesting compromise to express the modeled expressions at boundaries. A modified 3D-NSCBC strategy is discussed, in which the characteristic wave amplitude propagation is expressed in the frame attached to the flow streamline, to avoid the development, and eventually ease the evacuation, of spurious acoustic perturbations generated at boundaries. Kim \& Lee [12] also introduced change of coordinate system to apply NSCBC treatment for complex geometry outlets. However, their change of coordinate system was linked to the local geometry, not to the local streamline. For sake of brevity and to facilitate reading, only rectangular computational domains and cartesian orthonormal vector basis are considered, but the method can readily be generalized to any coordinates.

The following test cases are considered: an outgoing vortex with a mainstream that is oblique relative to the outlet boundary and an expanding 2D laminar flame, with a curved reactive front approaching a corner. Finally, the particular problem of an outgoing vortex flowing through a corner is analyzed. This last canonical problem combines the difficulty arising from interaction of two outgoing flows, inducing two ingoing waves close to the corner.

The paper is organized as follows. Section 2 states the governing equations in primitive variables formulation and its characteristic decomposition. It also briefly reminds how non reflecting outflows are handled by 3D-NSCBC. In section 3, limitations of NSCBC and 3D-NSCBC for non reflecting outflows are evidenced for two test cases. Section 4 further analyses these shortcomings by interpreting 3D-NSCBC as an acoustic forcing acting on the fluid that is not frame independent. In the light of these results, some improvements are proposed to capture the pertinent local acceleration felt by the fluid, leading to a better prediction of flow crossing boundaries.

\section{Characteristic equations for compressible flows}

\subsection{Primitive variables formulation}

Primitive variables formulation of multi-component Navier-Stokes equations may be cast in vector form as $\left(N_{\text {dim }}=2\right.$ or 3 space dimensions):

$$
\begin{aligned}
& \frac{\partial \widehat{\mathbf{Q}}}{\partial t}+\sum_{j=1}^{N_{d i m}} \widehat{\mathbf{d}}^{j}=\widehat{\mathbf{D}}+\widehat{\mathbf{S}} \\
& \widehat{\mathbf{d}}^{j}=\left(\begin{array}{l}
\widehat{d}_{i}^{j}=\widehat{U}_{j} \frac{\partial \widehat{U}_{i}}{\partial \widehat{x}_{j}}+\frac{1}{\rho} \frac{\partial P}{\partial \widehat{x}_{j}} \widehat{\delta}_{i j} \\
\widehat{d}_{4}^{j}=\frac{\partial \rho \widehat{U}_{j}}{\partial \widehat{x}_{j}} \\
\widehat{d}_{5}^{j}=\gamma P \frac{\partial \widehat{U}_{j}}{\partial \widehat{x}_{j}}+\widehat{U}_{j} \frac{\partial P}{\partial \widehat{x}_{j}} \\
\widehat{d}_{k}^{j}=\widehat{U}_{j} \frac{\partial Y_{k}}{\partial \widehat{x}_{j}}
\end{array}\right) \\
& \widehat{\mathbf{Q}}=\left(\begin{array}{c}
\widehat{Q}_{i}=\widehat{U}_{i} \\
\widehat{Q}_{4}=\rho \\
\widehat{Q}_{5}=P \\
\widehat{Q}_{k}=Y_{k}
\end{array}\right), \widehat{\mathbf{S}}=\left(\begin{array}{c}
\widehat{S}_{i} \\
0 \\
\widehat{S}_{5} \\
\widehat{S}_{k}
\end{array}\right)
\end{aligned}
$$

$$
\widetilde{\mathbf{d}}^{j}=\left(\begin{array}{l}
\frac{\partial \widetilde{\mathbf{Q}}}{\partial t}+\sum_{j=1}^{N_{\operatorname{dim}}} \widetilde{\mathbf{d}}^{j}=\widetilde{\mathbf{D}}+\widetilde{\mathbf{S}} \\
\widetilde{d}_{i}^{j}=\widetilde{U}_{j} \frac{\partial \widetilde{U}_{i}}{\partial \widetilde{x}_{j}}+\frac{1}{\rho} \frac{\partial \rho}{\partial \widetilde{U}_{j}} \frac{\partial P}{\partial \widetilde{x}_{j}} \widetilde{\delta}_{i j} \\
\widetilde{d_{5}^{j}}=\gamma P \frac{\partial \widetilde{U}_{j}}{\partial \widetilde{x}_{j}}+\widetilde{U}_{j} \frac{\partial P}{\partial \widetilde{x}_{j}} \\
\widetilde{\mathbf{Q}}=\left(\begin{array}{c}
\widetilde{d}_{k}^{j}=\widetilde{U}_{j} \frac{\partial Y_{k}}{\partial \widetilde{x}_{j}} \\
\widetilde{Q}_{i}=\widetilde{U}_{i} \\
\widetilde{Q}_{4}=\rho \\
\widetilde{Q}_{5}=P \\
\widetilde{Q}_{k}=Y_{k}
\end{array}\right), \quad \widetilde{\mathbf{S}}=\left(\begin{array}{c}
\widetilde{S}_{i} \\
0 \\
\widetilde{S}_{5} \\
\widetilde{S}_{k}
\end{array}\right)
\end{array}\right.
$$




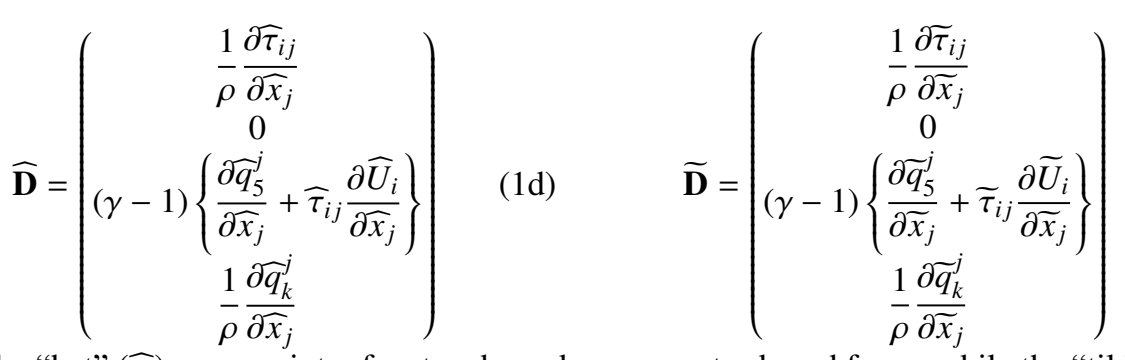

where the "hat" ( $\widetilde{.})$ superscript refers to a boundary geometry based frame while the "tilde" ( $\widetilde{.})$ superscript refers to the local streamline direction based frame. In the frame $\widehat{\mathcal{R}}=\left(O, \widehat{\boldsymbol{x}}_{1}, \widehat{\boldsymbol{x}}_{2}, \widehat{\boldsymbol{x}}_{3}\right)$, the components of the velocity vector $\mathbf{U}$ are denoted $\widehat{U}_{i}$ (for $i \in \llbracket 1, N_{\text {dim }} \rrbracket$ ) while they are denoted $\widetilde{U}_{i}$ (for $\left.i \in \llbracket 1, N_{\text {dim }} \rrbracket\right)$ in $\widetilde{\mathcal{R}}=\left(O, \widetilde{\boldsymbol{x}}_{1}, \widetilde{\boldsymbol{x}}_{2}, \widetilde{\boldsymbol{x}}_{3}\right)$. $\widehat{\mathbf{Q}}$ and $\widetilde{\mathbf{Q}}$ are the primitive variable vectors of velocity components $\widehat{U}_{i}$ and $\widetilde{U}_{i}$ (for $i \in \llbracket 1, N_{d i m} \rrbracket$ ), density $\rho$, pressure $P$ and mass fractions $Y_{k}$, $k \in \llbracket 1, N_{s} \rrbracket\left(N_{s}\right.$ the number of species). Vectors $\widetilde{\mathbf{d}}^{j}, \widehat{\mathbf{D}}$ and $\widehat{\mathbf{S}}$ (or $\widetilde{\mathbf{d}}^{j}, \widetilde{\mathbf{D}}$ and $\widetilde{\mathbf{S}}$ ) respectively represent convective (and pressure for velocity), diffusive and source terms. Using usual notation, it is assumed that $\widehat{\tau}_{i j}=\mu\left(\partial \widehat{U}_{i} / \partial \widehat{x}_{j}+\partial \widehat{U}_{j} / \partial \widehat{x}_{i}\right)-(2 / 3) \mu \operatorname{div} \widehat{\mathbf{U}} \widehat{\delta}_{i j} ; \mu=\mu_{o}\left(T / T_{o}\right)^{0.76} ; \widehat{q}_{5}^{j}=\lambda \partial T / \partial \widehat{x}_{j}$; $\widehat{q}_{k}^{j}=D \partial Y_{k} / \partial \widehat{x}_{j} ; \lambda=\mu C_{p} / \operatorname{Pr}$ and $D=\mu / S c_{k}$ (the reader may replace the 'hat' superscript by the 'tilde' superscript to get $\widetilde{\tau}_{i j}, \widetilde{q}_{5}^{j}, \widetilde{q}_{k}^{j}$ formulas). In following non-reactive cases, all source terms are zeros. In reactive cases, $\widehat{S}_{i}$ and $\widetilde{S}_{i}$ are zero while $\widehat{S}_{5}=\widetilde{S}_{5}$ and $\widehat{S}_{k}=\widetilde{S}_{k}$ are given by an Arrhenius law.

From rotational invariance, these conservation equations are formally the same by changing the coordinate system. However, as it shall be precised below, boundary condition modeling based on characteristic formulation is not frame independent. Next subsection details the modeled characteristic formulations for these two different frames. The variables expressed in the reference coordinate system used for computing discretized equations are without superscript.

\subsection{Characteristic variables formulation}

Convective terms $\widehat{d_{I}^{j}}$ can be expressed from advective terms of characteristic variables formulation $[1,2,5,13,14]$, as given by systems (3) and (5). By substituting $\widehat{\mathcal{L}}_{I}^{j}$ expressions into $\widehat{d}_{I}^{j}$ ones, one can recover equation (1b) for convective terms. The same characteristic formulation can be written in the local streamline direction based frame $\widetilde{\mathcal{R}}$, due to the rotational invariance.

$$
\begin{aligned}
\widehat{d}_{i=j}^{j} & =\frac{\widehat{\mathcal{L}}_{5}^{j}-\widehat{\mathcal{L}}_{4}^{j}}{2 \rho c} \\
\widehat{d}_{i \neq j}^{j} & =\widehat{\mathcal{L}}_{i}^{j} \\
\widehat{d}_{4}^{j} & =\frac{\widehat{\mathcal{L}}_{j}^{j}+\widehat{d}_{5}^{j}}{c^{2}} \\
\widehat{d}_{5}^{j} & =\frac{\widehat{\mathcal{L}}_{5}^{j}+\widehat{\mathcal{L}}_{4}^{j}}{2} \\
{\widehat{d_{k}^{j}}}^{j} & =\widehat{\mathcal{L}}_{k}^{j}
\end{aligned}
$$

$$
\begin{aligned}
\widetilde{d}_{i=j}^{j} & =\frac{\widetilde{\mathcal{L}}_{5}^{j}-\widetilde{\mathcal{L}}_{4}^{j}}{2 \rho c} \\
\widetilde{d}_{i \neq j}^{j} & =\widetilde{\mathcal{L}}_{i}^{j} \\
\widetilde{d}_{4}^{j} & =\frac{\widetilde{\mathcal{L}}_{j}^{j}+\widetilde{d}_{5}^{j}}{c^{2}} \\
\widetilde{d}_{5}^{j} & =\frac{\widetilde{\mathcal{L}}_{5}^{j}+\widetilde{\mathcal{L}}_{4}^{j}}{2} \\
\widetilde{d}_{k}^{j} & =\widetilde{\mathcal{L}}_{k}^{j}
\end{aligned}
$$




$$
\begin{aligned}
\widehat{\mathcal{L}}_{i=j}^{j} & =\widehat{U}_{j}\left(c^{2} \frac{\partial \rho}{\partial \widehat{x}_{j}}-\frac{\partial P}{\partial \widehat{x}_{j}}\right) \\
\widehat{\mathcal{L}}_{i \neq j}^{j} & =\widehat{U}_{j} \frac{\partial \widehat{U}_{i}}{\partial \widehat{x}_{j}} \\
\widehat{\mathcal{L}}_{4}^{j} & =\left(\widehat{U}_{j}-c\right)\left(\frac{\partial P}{\partial \widehat{x}_{j}}-\rho c \frac{\partial \widehat{U}_{j}}{\partial \widehat{x}_{j}}\right) \\
\widehat{\mathcal{L}}_{5}^{j} & =\left(\widehat{U}_{j}+c\right)\left(\frac{\partial P}{\partial \widehat{x}_{j}}+\rho c \frac{\partial \widehat{U}_{j}}{\partial \widehat{x}_{j}}\right) \\
\widehat{\mathcal{L}}_{k}^{j} & =\widehat{U}_{j} \frac{\partial Y_{k}}{\partial \widehat{x}_{j}}
\end{aligned}
$$

$$
\begin{aligned}
& \widetilde{\mathcal{L}}_{i=j}^{j}=\widetilde{U}_{j}\left(c^{2} \frac{\partial \rho}{\partial \widetilde{x}_{j}}-\frac{\partial P}{\partial \widetilde{x}_{j}}\right) \\
& \widetilde{\mathcal{L}}_{i \neq j}^{j}=\widetilde{U}_{j} \frac{\partial \widetilde{U}_{i}}{\partial \widetilde{x}_{j}}
\end{aligned}
$$$$
\widetilde{\mathcal{L}}_{4}^{j}=\left(\widetilde{U}_{j}-c\right)\left(\frac{\partial P}{\partial \widetilde{x}_{j}}-\rho c \frac{\partial \widetilde{U}_{j}}{\partial \widetilde{x}_{j}}\right)
$$

$$
\begin{aligned}
& \widetilde{\mathcal{L}}_{5}^{j}=\left(\widetilde{U}_{j}+c\right)\left(\frac{\partial P}{\partial \widetilde{x}_{j}}+\rho c \frac{\partial \widetilde{U}_{j}}{\partial \widetilde{x}_{j}}\right) \\
& \widetilde{\mathcal{L}}_{k}^{j}=\widetilde{U}_{j} \frac{\partial Y_{k}}{\partial \widetilde{x}_{j}}
\end{aligned}
$$

As already mentioned, we shall denote $\widetilde{\mathbf{x}}_{i}$ the basis vectors based on the local streamline direction. We choose $\widetilde{\mathbf{x}}_{1}=\mathbf{U} / U$, with $\mathbf{U}$ the local velocity. This defines a frame $\widetilde{\mathcal{R}}$. The basis vectors based on the geometry of the local boundary (defining a frame $\widehat{\mathcal{R}}$ ), are denoted $\widehat{\mathbf{x}}_{i}$, as shown in figure 1 . Vector $\widehat{\mathbf{x}}_{1}$ is the outgoing normal to the computational domain.

The calculation of derivatives in the $\widetilde{\mathcal{R}}$ frame is straightforward, once the gradient vector $\boldsymbol{\nabla} \phi$ of any $\phi$ variable is known in the computational frame $\mathcal{R}$,

$$
\frac{\partial \phi}{\partial \widetilde{x}_{i}}=\boldsymbol{\nabla} \phi \cdot \widetilde{\mathbf{x}}_{i}
$$

For instance, the velocity derivative $\partial \widetilde{U}_{2} / \partial \widetilde{x}_{1}$ may be computed from derivatives $\partial U_{i} / \partial x_{j}$ estimated with a sixth order collocated Padé scheme [15] and from coordinates $\widetilde{x}_{j 1}$ and $\widetilde{x}_{i 2}$ of vectors $\widetilde{\mathbf{x}}_{1}$ and $\widetilde{\mathbf{x}}_{2}$ in $\mathcal{R}$ :

$$
\frac{\partial \widetilde{U}_{2}}{\partial \widetilde{x}_{1}}=\nabla\left(\mathbf{U} \cdot \widetilde{\mathbf{x}}_{\mathbf{2}}\right) \cdot \widetilde{\mathbf{x}}_{\mathbf{1}}=\sum_{k=1}^{N_{\text {dim }}} \sum_{l=1}^{N_{d i m}} \widetilde{x}_{i 2} \frac{\partial U_{i}}{\partial x_{j}} \widetilde{x}_{j 1}
$$

For simplicity a two-dimensional cartesian grid is used with frame $\mathcal{R}$, but extension of present methodology to three space dimensions is straightforward. If the outflow boundary is constituted by a unique face or plane, it is further assumed that this frame coincides (up to $\pm \pi / 2$ rotations)

a)

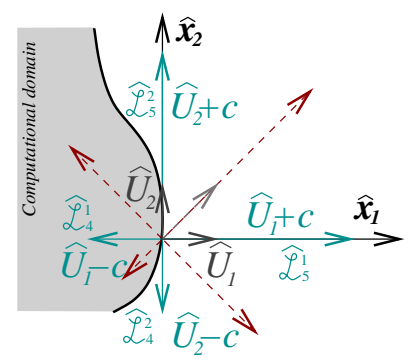

b)

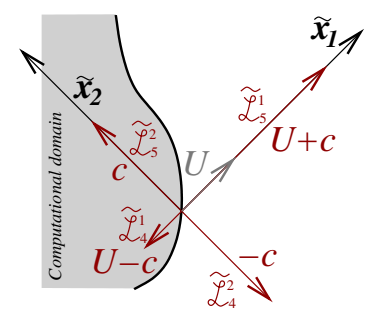

Figure 1: Schematic of characteristic wave projections in two coordinate systems: Left, geometry based frame $\widehat{\mathcal{R}}$. Right, local streamline based frame $\widetilde{\mathcal{R}}$. The grey area is the computational domain and the dark line its boundary. 
with the previously introduced geometry based frame $\widehat{\mathcal{R}}$. This may not be true for instance at outflowing corners and/or edges, when there is locally more than one outflowing face (see subsections 4.3 and 4.4). We then choose to define the local normal vector at a corner (or an edge) as the mean of the normal vectors to the adjacent facets to the node. In this case, local geometry based frame $\widehat{\mathcal{R}}$ and the computing reference system of coordinates $\mathcal{R}$ may differ.

For the sake of clarity, figure 1 shows a $2 \mathrm{D}$ scheme of possible characteristic wave projections, yielding to different boundary condition treatment procedures.

In a first vision (see figure 1a), adopted by NSCBC and linked to the geometry, characteristic wave amplitudes propagate along the two directions defined by vectors $\widehat{\mathbf{x}}_{\mathbf{1}}$ and $\widehat{\mathbf{x}}_{\mathbf{2}}$. Entropy $\left(\widehat{\mathcal{L}}_{i=j}^{j}\right)$ and transverse velocity $\left(\widehat{\mathcal{L}}_{i \neq j}^{j}\right)$ characteristic waves are convected at speeds $\widehat{U}_{1}$ and $\widehat{U}_{2}$, along basis vectors $\widehat{\mathbf{x}}_{\mathbf{1}}$ and $\widehat{\mathbf{x}}_{\mathbf{2}}$. Acoustic characteristics of amplitudes $\widehat{\mathcal{L}}_{4}^{j}$ and $\widehat{\mathcal{L}}_{5}^{j}$ propagate towards $\widehat{\mathbf{x}}_{\mathbf{j}}$ directions with respectively $\widehat{U}_{j}-c$ and $\widehat{U}_{j}+c$ velocities.

In a second vision (see figure $1 \mathrm{~b}$ ), tentatively adopted in the present work, characteristics now propagate in directions defined by the local velocity of the flow. Entropy $\left(\widetilde{\mathcal{L}}_{i=j}^{j}\right)$ and transverse velocity $\left(\widetilde{\mathcal{L}}_{i \neq j}^{j}\right)$ waves are advected at speed $\widetilde{U}_{1} \equiv U$, along $\widetilde{\mathbf{x}}_{\mathbf{1}}$. In local flow direction, acoustic waves $\left(\widetilde{\mathcal{L}}_{4}^{1}\right.$ and $\left.\widetilde{\mathcal{L}}_{5}^{1}\right)$ propagate at $U-c$ and $U+c$. In the direction normal to the flow $\widetilde{\mathbf{x}}_{\mathbf{2}}, \widetilde{\mathcal{L}}_{4}^{2}$ and $\widetilde{\mathcal{L}}_{5}^{2}$ propagate respectively at $+c$ and $-c$.

Wave amplitudes $\widehat{\mathcal{L}}_{I}^{j}$ or $\widetilde{\mathcal{L}}_{I}^{j}$ may be expressed in frame $\widehat{\mathcal{R}}$ or $\widetilde{\mathcal{R}}$ whilst primitive variables conservation are expressed in frame $\mathcal{R}$. Convective terms $d_{i}=\sum_{j=1}^{N_{\text {dim }}} d_{i}^{j}$ in $\mathcal{R}$ may be expressed as functions of convective terms $\widehat{d_{i}}=\sum_{j=1}^{N_{\text {dim }}} \widehat{d_{i}^{j}}$ or $\widetilde{d_{i}}=\sum_{j=1}^{N_{\text {dim }}} \widetilde{d}_{i}^{j}$ and wave amplitudes $\widehat{\mathcal{L}}_{I}^{j}$ or $\widetilde{\mathcal{L}}_{I}^{j}$, as

$$
\begin{array}{lll}
d_{1}=\widehat{d}_{1} \widehat{x}_{11}+\widehat{d}_{2} \widehat{x}_{12}=\frac{\widehat{\mathcal{L}}_{5}^{j}-\widehat{\mathcal{L}}_{4}^{j}}{2 \rho c} \widehat{x}_{1 j}+\widehat{\mathcal{L}}_{1}^{2} \widehat{x}_{11}+\widehat{\mathcal{L}}_{2}^{1} \widehat{x}_{12}=\frac{\widetilde{\mathcal{L}}_{5}^{j}-\widetilde{\mathcal{L}}_{4}^{j}}{2 \rho c} \widetilde{x}_{1 j}+\widetilde{\mathcal{L}}_{1}^{2} \widetilde{x}_{11}+\widetilde{\mathcal{L}}_{2}^{1} \widetilde{x}_{12} \\
d_{2}=\widehat{d}_{1} \widehat{x}_{21}+\widehat{d}_{2} \widehat{x}_{22}=\frac{\widehat{\mathcal{L}}_{5}^{j}-\widehat{\mathcal{L}}_{4}^{j}}{2 \rho c} \widehat{x}_{2 j}+\widehat{\mathcal{L}}_{1}^{2} \widehat{x}_{21}+\widehat{\mathcal{L}}_{2}^{1} \widehat{x}_{22} & =\frac{\widetilde{\mathcal{L}}_{5}^{j}-\widetilde{\mathcal{L}}_{4}^{j}}{2 \rho c} \widehat{x}_{2 j}+\widetilde{\mathcal{L}}_{1}^{2} \widetilde{x}_{21}+\widetilde{\mathcal{L}}_{2}^{1} \widetilde{x}_{22} \\
d_{4}=\widehat{d}_{4} & =\frac{1}{c^{2}}\left(\widehat{\mathcal{L}}_{1}^{1}+\widehat{\mathcal{L}}_{2}^{2}+d_{5}\right) & =\frac{1}{c^{2}}\left(\widetilde{\mathcal{L}}_{1}^{1}+\widetilde{\mathcal{L}}_{2}^{2}+d_{5}\right) \\
d_{5}=\widehat{d}_{5} & =\frac{1}{2}\left(\widehat{\mathcal{L}}_{4}^{1}+\widehat{\mathcal{L}}_{5}^{1}+\widehat{\mathcal{L}}_{4}^{2}+\widehat{\mathcal{L}}_{5}^{2}\right) & =\frac{1}{2}\left(\widetilde{\mathcal{L}}_{4}^{1}+\widetilde{\mathcal{L}}_{5}^{1}+\widetilde{\mathcal{L}}_{4}^{2}+\widetilde{\mathcal{L}}_{5}^{2}\right) \\
d_{k}=\widehat{d}_{k} & =\widehat{\mathcal{L}}_{k}^{1}+\widehat{\mathcal{L}}_{k}^{2} & =\widetilde{\mathcal{L}}_{k}^{1}+\widetilde{\mathcal{L}}_{k}^{2}
\end{array}
$$

$\widehat{x}_{i j}$ and $\widetilde{x}_{i j}$ being the $i$-th coordinate of vectors $\widehat{\mathbf{x}}_{\mathbf{j}}$ and $\widetilde{\mathbf{x}}_{\mathbf{j}}$ in the $\mathcal{R}$ coordinate frame. The term,

$$
d_{5}=\frac{1}{2} \sum_{j=1}^{N_{\text {dim }}}\left(\widehat{\mathcal{L}}_{4}^{j}+\widehat{\mathcal{L}}_{5}^{j}\right)=\frac{1}{2} \sum_{j=1}^{N_{\text {dim }}}\left(\widetilde{\mathcal{L}}_{4}^{j}+\widetilde{\mathcal{L}}_{5}^{j}\right)=\mathbf{U} \cdot \boldsymbol{\nabla} P+\rho c^{2} \boldsymbol{\nabla} P \cdot \mathbf{U}
$$

is clearly coordinate system independent, as $\sum_{j=1}^{N_{\text {dim }}} \widehat{\mathcal{L}}_{j}^{j}=\sum_{j=1}^{N_{\text {dim }}} \widetilde{\mathcal{L}}_{j}^{j}=c^{2} \mathbf{U} \cdot \boldsymbol{\nabla} \rho-\mathbf{U} \cdot \boldsymbol{\nabla} P, d_{4}=\widehat{d}_{4}=$ $\widetilde{d}_{4}=\boldsymbol{\nabla} \cdot(\rho \mathbf{U}), d_{5}=\widehat{d}_{5}=\widetilde{d}_{5}=\gamma P \boldsymbol{\nabla} \cdot \mathbf{U}+\mathbf{U} \cdot \boldsymbol{\nabla} P$ and $d_{k}=\widehat{d}_{k}=\widetilde{d}_{k}=\mathbf{U} \cdot \boldsymbol{\nabla} Y_{k}$. The acceleration in 
the inviscid context may be written:

$$
\frac{\partial \mathbf{U}}{\partial t}=-\sum_{j=1}^{N_{\text {dim }}} \frac{\widetilde{\mathcal{L}}_{5}^{j}-\widetilde{\mathcal{L}}_{4}^{j}}{2 \rho c} \widetilde{\boldsymbol{x}}_{j}-\sum_{\substack{j=1 \\ j \neq i}}^{N_{\text {dim }}} \widetilde{\mathcal{L}}_{i}^{j} \widetilde{\boldsymbol{x}}_{i}
$$

thus

$$
\sum_{j=1}^{N_{\text {dim }}} \frac{\widetilde{\mathcal{L}}_{5}^{j}-\widetilde{\mathcal{L}}_{4}^{j}}{2 \rho c} \widetilde{\boldsymbol{x}}_{j}=(\mathbf{U} \cdot \boldsymbol{\nabla}) \mathbf{U}+\frac{1}{\rho} \boldsymbol{\nabla} P-\sum_{\substack{j=1 \\ j \neq i}}^{N_{\text {dim }}} \widetilde{\mathcal{L}}_{i}^{j} \widetilde{\boldsymbol{x}}_{i}
$$

The LHS of (12) is usually modeled independently of the boundary plane contribution, $\sum_{j=1, j \neq i}^{N_{\text {dim }}} \widehat{\mathcal{L}}_{i}^{j} \widehat{\boldsymbol{x}}_{i}$ or $\sum_{j=1, j \neq i}^{N_{d i m}} \widetilde{\mathcal{L}}_{i}^{j} \widetilde{\boldsymbol{x}}_{i}$, whereas such terms do not have rotationaly invariant expressions. The impact of this observation on $\mathrm{BC}$ is now investigated.

\subsection{Summary of $3 D-N S C B C$ for non reflecting outflows}

In 3D-NSCBC [4, 5], for outflows, the coordinate system is aligned with the normal vector to the outflowing facet (or plane). As already mentionned, the variables relative to this geometry based frame are denoted with . superscript. For outflows, all wave amplitudes $\widehat{\mathcal{L}}_{I}^{j}$ (see equations (5)) are computed from the internal side of the computational domain, except the acoustic term $\widehat{\mathcal{L}}_{4}^{1}$, able to propagate at velocity $\widehat{U}_{1}-c$ along direction $\widehat{\mathbf{x}}_{\mathbf{1}}$ normal to the outflow plane. In [2], the amplitude $\widehat{\mathcal{L}}_{4}^{1}$ is specified by equation

$$
\widehat{\mathcal{L}}_{4}^{1}=\eta_{4}\left(P-P_{\infty}^{\text {out }}\right)
$$

The introduced coefficient $\eta_{4}$ allows to relax the outflow pressure towards a prescribed value $P_{\infty}^{\text {out }}$. Yoo et al. [3] and Lodato et al. [5] included some additional transverse terms:

$$
\widehat{\mathcal{L}}_{4}^{1}=\eta_{4}\left(P-P_{\infty}^{\text {out }}\right)+\widehat{\eta}_{1}\left(\widehat{\mathbb{T}}_{4}^{\text {out }}-\widehat{\mathbb{T}}_{4}\right)+\widehat{\mathbb{T}}_{4}
$$

Thanks to an asymptotic Low Mach analysis [3], the relaxation coefficient $\widehat{\eta}_{1}$ is chosen equal to the local mean Mach number $\widehat{U}_{1} / c$. The $\widehat{\mathbb{T}}_{4}^{\text {out }}$ term analyticaly prescribes the transverse terms at the outflow. Yoo \& Im [4] complete this treatment by adding diffusive and reactive transverse terms :

$$
\begin{aligned}
& \widehat{\mathcal{L}}_{4}^{1}=\eta_{4}\left(P-P_{\infty}^{\text {out }}\right)+\widehat{\eta}_{1}\left(\widehat{\mathbb{T}}_{4}^{\text {out }}-\widehat{\mathbb{T}}_{4}\right)+\widehat{\mathbb{T}}_{4}+\widehat{\mathbb{D}}_{4}+\widehat{\mathbb{S}}_{4} \\
& \widehat{\mathbb{T}}_{4}=-\sum_{j=2}^{N_{\text {dim }}} \widehat{d}_{5}^{j}-\rho c \widehat{d}_{1}^{j} \\
& \widehat{\mathbb{D}}_{4}=D_{5}-\rho c \widehat{D}_{1} \\
& \widehat{\mathbb{S}}_{4}=S_{5}-\rho c \widehat{S}_{1}
\end{aligned}
$$

\section{Shortcomings of NSCBC and 3D-NSCBC treatments}

Two canonical test cases are now discussed to illustrate specific perturbations appearing with NSCBC and 3D-NSCBC treatments in the case of oblique flows at boundaries. The sixth order accurate in space and third order in time Direct Numerical Simulation code 'Allegro' [16] is used with a single-step chemistry cast as in [17]. In all test cases performed in this article, the time step is set by a CFL condition with $\Delta t=0.4 \cdot \max _{i} \Delta x_{i} /\left(\left|U_{i}\right|+c\right)$. 


\subsection{Laminar expanding premixed flame}

The test case proposed by Yoo and $\mathrm{Im}[4]$ is reproduced. A laminar 2D expanding stoichiometric premixed flame is computed over a $38 \delta_{L} \times 38 \delta_{L}$ square domain with 200 mesh points in every direction. In the reactive flow case, the characteristic flame speed and thickness $\delta_{L}$ are used to make results dimensionless. All BCs (including corners) are non reflecting outflows and the zero viscous flux conditions at boundaries $[18,4]$ is used: $\partial \widehat{q}_{5}^{1} / \partial \widehat{x}_{1}=0, \partial \widehat{q}_{k}^{1} / \partial \widehat{x}_{1}=0$ and $\partial \widehat{\tau}_{21} / \partial \widehat{x}_{1}=0$.
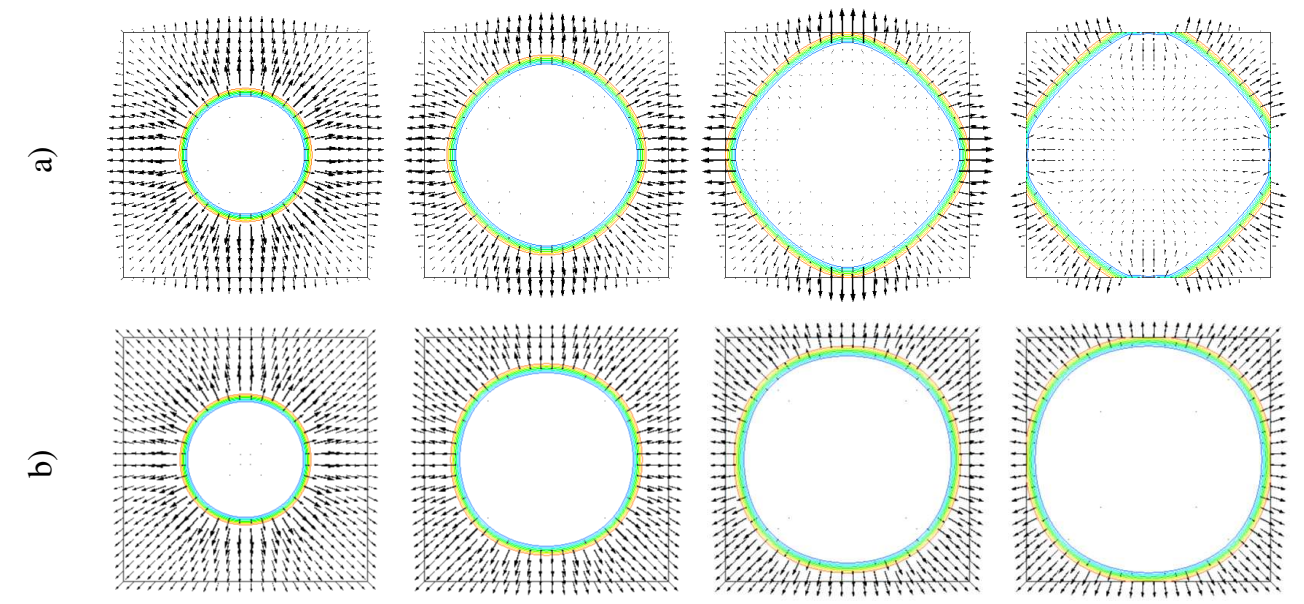

Figure 2: Expanding laminar flame: a) Fuel mass fraction iso-values and velocity vector field with NSCBC outflow; (b) same isovalues with 3D-NSCBC outflow treatment.

The difficulties arisen in this test case are mainly twofold: i) the front is a thin reactive zone, involving diffusive and reactive terms that should be accounted for close to the boundaries ${ }^{1}$; ii) since the flow is isotropically expanding, local velocity directions are diverging from the local normal to the boundary facets. Figure 2 shows fuel mass fraction and velocity vectors evolution, for respectively NSCBC and 3D-NSCBC outflow treatment. With "standard" NSCBC outflow [2], as shown in figure 2a), the flame shape tends to become square when reaching the boundaries. Velocity decreases at corners, and the velocity field is not uniformly expanding. Only when velocity components are almost normal to the boundaries of the computational domain is the NSCBC treatment efficient. For 3D-NSCBC [4,5], the flame remains more circular, even if its shape clearly tends to become square in the end (see figure $2 b$ ). In [4], the authors suggest this behavior is mainly due to the relaxing pressure term $\eta_{4}\left(P-P_{\infty}^{\text {out }}\right)$. However, even when $\eta_{4}=0$ (cf. figure 2), a square-shaped behavior is still found close to the boundaries.

\subsection{Outgoing vortex}

A $2 \mathrm{D}$ vortex is convected from left to right and exits the square $10 R_{V} \times 10 R_{V}$ computational domain. The initial condition (16), taken from a potential flow solution [5, 19], is a solution of the Euler equations. This isothermal vortex test case is a well known test for boundary conditions [4];

\footnotetext{
${ }^{1}$ Taking into account diffusive $\widehat{\mathbb{D}}_{4}$ and reactive $\widehat{\mathbb{S}}_{4}$ terms (see equation (15a)) is crucial, by omitting these terms, the computation becomes unstable at domain corners.
} 
it has been retained since it often reveals spurious distortions at boundaries due to the presence of transverse terms that are difficult to handle in the Euler or Navier-Stokes equations.

$$
\begin{aligned}
& P(R)=P_{o} \cdot \exp ^{\left[-\frac{\gamma}{2}\left(\frac{C_{V}}{c R_{V}}\right)^{2} \cdot \exp ^{-\left(\frac{R}{R_{V}}\right)^{2}}\right]} \\
& \rho(R)=\frac{P(R)}{r T_{o}} \\
& \mathbf{U}(R)=\underbrace{C_{V} \frac{R}{R_{V}^{2}} \cdot \exp ^{-\frac{1}{2}\left(\frac{R}{R_{V}}\right)^{2}}}_{U_{\theta}(R)} \overrightarrow{e_{\theta}}+\widehat{U}_{1}^{i n} \widehat{\boldsymbol{x}}_{\mathbf{1}}+\widehat{U}_{2}^{i n} \widehat{\boldsymbol{x}}_{\mathbf{2}}
\end{aligned}
$$

where $\overrightarrow{e_{\theta}}$ is the ortho-radial vector of the cylindrical basis. In the outgoing vortex case, the vortex radius $R_{V}$ and the sound velocity $c$ are used as scaling parameters. The maximum tangential velocity is denoted by $U_{\theta}^{m}=U_{\theta}\left(R_{V}\right)$. The mesh is composed of $100 \times 100$ grid points and fluid is atmospheric air. Right BC (including corners) is non reflecting outflow, while up and down faces are periodic. A mean flow (the mainstream) normal to the right outlet face - the normal direction of which being indexed as $1-$ is imposed : $\widehat{U}_{1}^{i n} / c=0.1$ and $\widehat{U}_{2}^{i n}=0$. To

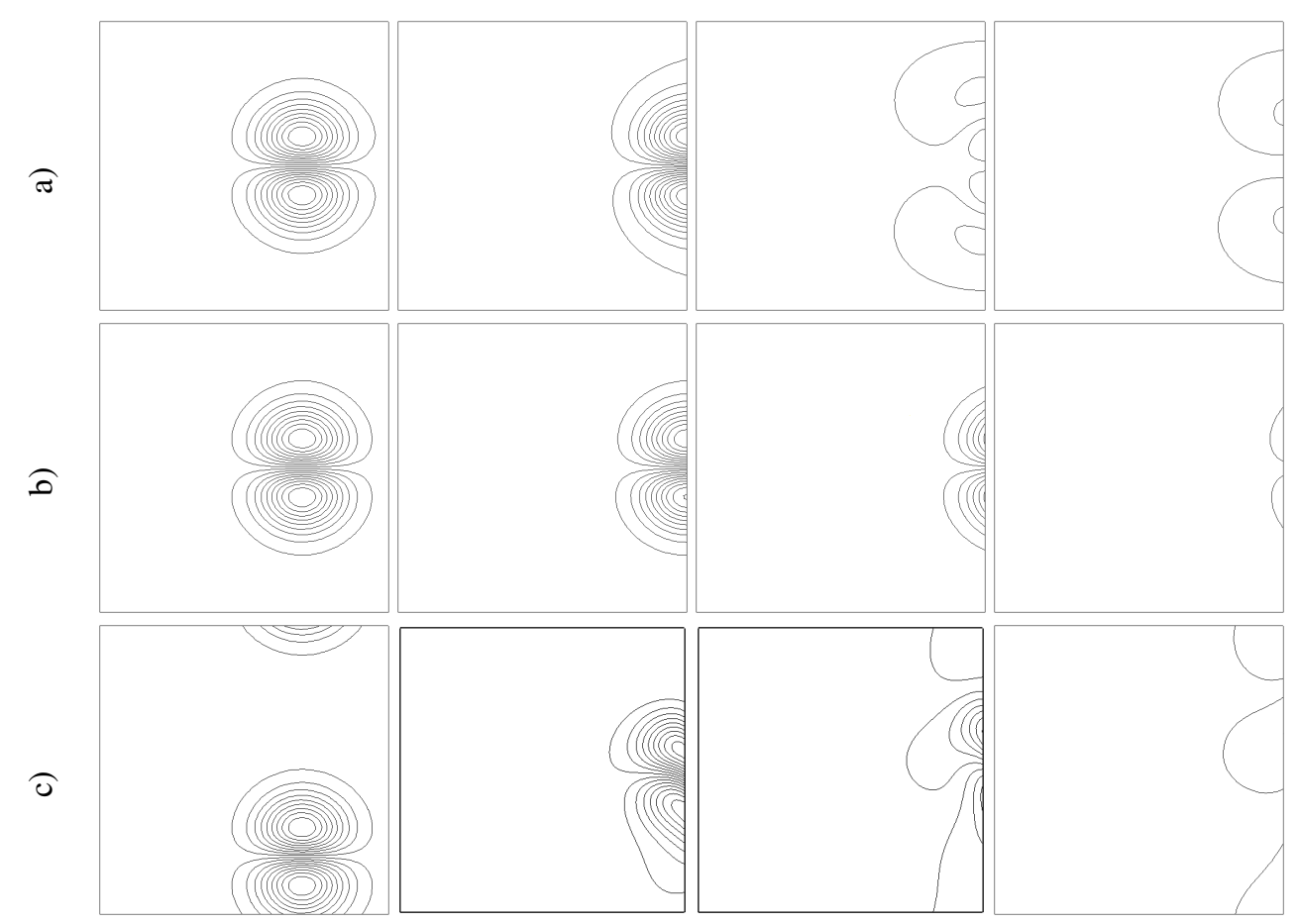

Figure 3: Outgoing vortex (see [5]) computations $\left(U_{1}^{i n} / c=0.1\right.$ and $U_{\theta}^{m} / c=0.0066$ ). a) iso- $U_{1}$, "standard" NSCBC outflow of [2]; b) iso- $U_{1}, 3 \mathrm{D}-\mathrm{NSCBC}$ outflow treatment $[4,5]$, mainstream normal to the outflow; c) iso- $U_{1}, 3 \mathrm{D}-\mathrm{NSCBC}$ outflow, mainstream has a transverse component.

evidence non physical acoustic reflection at the outflows when the mainstream is not normal to 
the computational boundaries, the same computation is done but with an oblique mean velocity such that $\widehat{U}_{1}^{i n} / c=\widehat{U}_{2}^{i n} / c=0.1$. The vortex intensity in both cases is such that $U_{\theta}^{m} / c=0.0066$.

Figure 3 shows velocity component $U_{1}$ when the vortex crosses the right boundaries, respectively with the normal or the oblique (at 45 degrees) mean velocity. For both calculations, $\widehat{\eta}_{1}=\widehat{U}_{1} / c$ and $\widehat{\mathbb{T}}_{4}^{\text {out }}=0$.

With a mean velocity normal to the right boundary (cf. figure 3b), as already reported in [5], the 3D-NSCBC pertinently lower acoustic reflection, compared with a standard NSCBC (figure 3a). However, when the mainstream is not normal to the right boundary (figure 3c), the vortex is significantly warped at outlet vicinity. This behavior does not change for weaker vortex intensities, or for other values of the relaxation coefficient $\widehat{\eta}_{1}$ (taking e.g. $U / c$, with $U$ local velocity norm at outlet, $\max U / c, \max \widehat{U}_{1} / c$ or even parametric arbitrary values). Hence, flow acceleration at the boundary seems not perfectly modeled by 3D-NSCBC when the mainstream is no more normal to the boundary.

\section{Transverse flow non reflecting outlet}

\subsection{Modeled acoustic force}

The physics described by systems (3) or (4) should be independent of the chosen coordinate system. In fact, we shall demonstrate in the sequel that the choice of a particular coordinate system becomes crucial as soon as some amplitudes $\widehat{\mathcal{L}}_{I}^{j}$ or $\widetilde{\mathcal{L}}_{I}^{j}$ are modeled.

The conservation equations for momentum in (1) or (2) may be re-written as

$$
\begin{array}{r}
\rho \frac{d \widehat{U}_{1}}{d t}+\frac{\partial P}{\partial \widehat{x}_{1}}=\frac{\partial \widehat{\tau}_{i j}}{\partial \widehat{x}_{j}}+\rho \widehat{S}_{1}+\overbrace{\frac{1}{2 c}\left\{\widehat{\mathcal{L}}_{4}^{1}-\left(\widehat{U}_{1}-c\right)\left(\frac{\partial P}{\partial \widehat{x}_{1}}-\rho c \frac{\left.\partial \widehat{U}_{1}\right)}{\partial \widehat{x}_{1}}\right)\right\}}^{\widehat{f}_{a c}} \\
\rho \frac{d \widetilde{U}_{1}}{d t}+\frac{\partial P}{\partial \widetilde{x}_{1}}=\frac{\partial \widetilde{\tau}_{i j}}{\partial \widetilde{x}_{j}}+\rho \widetilde{S}_{1}+\underbrace{\frac{1}{2 c}\left\{\widetilde{\mathcal{L}}_{4}^{1}-\left(\widetilde{U}_{1}-c\right)\left(\frac{\partial P}{\partial \widetilde{x}_{1}}-\rho c \frac{\partial \widetilde{U}_{1}}{\partial \widetilde{x}_{1}}\right)\right\}}_{\widetilde{f}_{a c}}
\end{array}
$$

When the wave amplitude $\widehat{\mathcal{L}}_{4}^{1}$ (resp. $\widetilde{\mathcal{L}}_{4}^{1}$ ) can be computed by its analytic expression (6c), i.e. inside the computational domain, the above term $\widehat{f}_{a c}$ (resp. $\widetilde{f}_{a c}$ ) - homogeneous to a volumic force - is zero. When this wave amplitude $\widehat{\mathcal{L}}_{4}^{1}$ is modeled by a 3D-NSCBC outflow boundary treatment, this non-zero term can be interpreted as a force (of acoustic origin) acting on the fluid from the outside of the domain. Substituting $\widehat{\mathcal{L}}_{4}^{1}$ (wave intensity expressed in the geometry based frame) by their modeled expressions $\left((13)\right.$ or (14) with $\eta_{4}=0, \widehat{\eta_{1}}=\widehat{U_{1}} / c$ and $\left.\widehat{\mathbb{T}}_{4}^{\text {out }}=0\right)$, into equation (17a), we obtain respectively expressions (18a) and (18b) for the acoustic force $\widehat{f}_{a c}$. Note that this interpretation is also valid in $3 \mathrm{D}$.

These forces, denoted respectively as vectors $\widehat{\mathbf{f}_{\mathbf{a c}}^{\mathbf{0}}}$ and $\widehat{\mathbf{f}_{\mathbf{a c}}^{\mathbf{1}}}$, can be interpreted as models of acoustic forces, exerted from the exterior on the fluid, and added respectively by NSCBC modeling of [2] or 3D-NSCBC modeling [5]. Notice the presence of transverse terms in $\mathbf{f}_{\mathbf{a c}}^{\mathbf{1}}$ compared 
with $\widehat{\mathbf{f a c}_{\mathbf{a c}}^{0}}$

$$
\begin{aligned}
& \widehat{\mathbf{f}_{\mathbf{a c}}^{\mathbf{0}}}=\frac{1-\widehat{U}_{1} / c}{2}\left\{\frac{\partial P}{\partial \widehat{x}_{1}}-\rho c \frac{\partial \widehat{U}_{1}}{\partial \widehat{x}_{1}}\right\} \widehat{\mathbf{x}}_{\mathbf{1}} \\
& \widehat{\mathbf{f}_{\mathbf{a c}}^{\mathbf{1}}}=\frac{1-\widehat{U}_{1} / c}{2}\left\{\frac{\partial P}{\partial \widehat{x}_{1}}-\rho c \frac{\partial \widehat{U}_{1}}{\partial \widehat{x}_{1}}-\frac{\widehat{U}_{2}}{c}\left(\frac{\partial P}{\partial \widehat{x}_{2}}-\rho c \frac{\partial \widehat{U}_{1}}{\partial \widehat{x}_{2}}\right)-\rho c \frac{\partial \widehat{U}_{2}}{\partial \widehat{x}_{2}}\right\} \widehat{\mathbf{x}}_{\mathbf{1}}
\end{aligned}
$$

However, from the results of figure $3 \mathrm{c}$, when the mainstream is not normal to the outlet face, it seems that this $3 \mathrm{D}-\mathrm{NSCBC}$ approximated force $\widehat{\mathbf{f}_{\mathrm{ac}}^{1}}$ is not effectively able to correctly reproduce the "real" (physical) force that allows a vortex going through the domain with no deformation. It is thus anticipated that the asymptotic analysis of [4] shows that $\widetilde{\eta}_{1}=\widetilde{U}_{1} / c$ is a good choice for $\widetilde{\mathbb{T}}_{4}^{\text {out }}=0$, but solely when the reference frame is aligned with the direction of the local mainstream. With above notations, this would mean $\widetilde{\mathcal{R}} \equiv \widehat{\mathcal{R}}$ (see figure 1).

In the local streamline based frame $\widetilde{\mathcal{R}}$ where $\widetilde{U}_{2}\left(=\widetilde{U}_{3}\right)=0$ and also $\widetilde{\mathbb{T}}_{4}^{\text {out }}=0$, if we now subsitute $\widetilde{\mathcal{L}}_{4}^{1}$ into equation (17b) by its modeled expression

$$
\begin{aligned}
\widetilde{\mathcal{L}}_{4}^{1} & =\widetilde{\eta}_{1}\left(\widetilde{\mathbb{T}}_{4}^{\text {out }}-\widetilde{\mathbb{T}}_{4}\right)+\widetilde{\mathbb{T}}_{4}+\widetilde{\mathbb{D}}_{4}+\widetilde{\mathbb{S}}_{4} \\
\widetilde{\mathbb{T}}_{4} & =-\sum_{j=2}^{N_{\text {dim }}} \widetilde{d}_{5}^{j}-\rho c{\widetilde{d_{1}^{j}}}^{j} \\
\widetilde{\mathbb{D}}_{4} & =D_{5}-\rho c \widetilde{D}_{1} \\
\widetilde{\mathbb{S}}_{4} & =S_{5}-\rho c \widetilde{S}_{1}
\end{aligned}
$$

(with $\eta_{4}=0$ ), we obtain a new acoustic force, denoted $\widetilde{\mathbf{f}_{\mathbf{a c}}^{2}}$ :

$$
\widetilde{\mathbf{f}_{\mathbf{a c}}^{2}}=\frac{1-\widetilde{U}_{1} / c}{2}\left\{\frac{\partial P}{\partial \widetilde{x}_{1}}-\rho c \frac{\partial \widetilde{U}_{1}}{\partial \widetilde{x}_{1}}-\rho c \frac{\partial \widetilde{U}_{2}}{\partial \widetilde{x}_{2}}\right\} \widetilde{\mathbf{x}}_{\mathbf{1}}
$$

Note that transverse terms corresponding to $\widetilde{\mathbf{x}}_{\mathbf{2}}$ (and $\widetilde{\mathbf{x}}_{\mathbf{3}}$ ) vanish, and that this force is now parallel to $\widetilde{\mathbf{x}}_{\mathbf{1}}$. In these equations, the relation (7) is used in the solver to compute the derivatives in the streamline frame.

The $\widetilde{\mathcal{L}}_{4}^{1}$ is now calculated according to

$$
\widetilde{\mathcal{L}}_{4}^{1}=\left(1-\widetilde{U}_{1} / c\right)\left(-\gamma P \frac{\partial \widetilde{U}_{2}}{\partial \widetilde{x}_{2}}\right)
$$

in order to reproduce an acoustic force $\widetilde{\mathbf{f}_{\mathbf{a c}}^{2}}$ compatible with the asymptotic analysis of [4]. All other amplitudes $\widetilde{\mathcal{L}}_{I}^{j}$ are determined through their analytic expressions (6). We shall call this proposed boundary treatment "3D-NSCBC-TO", for Transverse Outflow. This last expression reduces to 3D-NSCBC in the case of a mainstream flow normal to the boundary face.

This attempt to adapt 3D-NSCBC outflow treatment was first tested in the case of a twodimensional outgoing vortex passing through a corner. Anticipating on subsections 4.2, 4.3 and figures 5, 4 and 9, where all methods are systematically compared, we observed a much more satisfying behavior than with 3D-NSCBC. The shape of the exiting vortex remains cylindrical at boundary vicinity, while it was significantly warped with 3D-NSCBC. However, we also 
observed a weak numerical instability after outlet crossing, indicating that 3D-NSCBC-TO modeling introduces errors in the $\widetilde{\mathbf{x}}_{\mathbf{2}}$ direction. This corner makes pressure, density and velocity to oscillate in the whole domain while a back-flow develops after the vortex has left the computational domain. When simulating an ignition kernel, these oscillations appear along outflows at the first iterations. This may be explained by the fact that the amplitude $\widetilde{L}_{4}^{2}$ should not be computed from the outside of the domain, since it brings information from the inside, as shown in figure 1 . This suggests to look for a numerically stabilized modification to this first attempt. It is hence proposed in a second step to compute all amplitudes $\widehat{\mathcal{L}}_{I}^{j}$ except $\widehat{\mathcal{L}}_{4}^{1}$. This last one is now specified in order to reproduce the same acceleration as with 3D-NSCBC-TO treatment along the normal vector $\widehat{\mathbf{x}}_{1}$ :

$$
\widehat{\mathcal{L}}_{4}^{1}=\widehat{\mathcal{L}}_{5}^{1}+2 \rho c \widehat{\mathcal{L}}_{1}^{2}-\left(\widetilde{\mathcal{L}}_{5}^{1}-\widetilde{\mathcal{L}}_{4}^{1}+2 \rho c \widetilde{\mathcal{L}}_{1}^{2}\right) \widetilde{\mathbf{x}}_{\mathbf{1}} \cdot \widehat{\mathbf{x}}_{\mathbf{1}}-\left(\widetilde{\mathcal{L}}_{5}^{2}-\widetilde{\mathcal{L}}_{4}^{2}+2 \rho c \widetilde{\mathcal{L}}_{2}^{1}\right) \widetilde{\mathbf{x}}_{\mathbf{2}} \cdot \widehat{\mathbf{x}}_{\mathbf{1}}
$$

Equation (22) can be obtained after projection of the acceleration vector $\partial \widetilde{\mathbf{U}} / \partial t$ along $\widehat{\mathbf{x}}_{\mathbf{1}}$ :

$$
\rho\left(\frac{\partial \widetilde{U}_{1}}{\partial t} \widetilde{\mathbf{x}}_{1}+\frac{\partial \widetilde{U}_{2}}{\partial t} \widetilde{\mathbf{x}}_{2}\right)=\rho\left(\frac{\partial \widehat{U}_{1}}{\partial t} \widehat{\mathbf{x}}_{1}+\frac{\partial \widehat{U}_{2}}{\partial t} \widehat{\mathbf{x}}_{2}\right)
$$

and by omitting reactive and diffusion terms in $\partial \widehat{U}_{i} / \partial t$ and $\partial \widetilde{U}_{i} / \partial t$ expressions. Note that if $\widetilde{\mathbf{x}}_{i} \equiv \widehat{\mathbf{x}}_{i}$, equation (22) yields again 3D-NSCBC.

This modified second proposed strategy shall be referred to as 3D-NSCBC-TOM, for Transverse Outflow Modified. Notice that 3D-NSCBC-TOM is introduced to suppress the instability of the 3D-NSCBC-TO treatment while modeling the same fluid acceleration in the $\widehat{\mathbf{x}}_{1}$ direction. To validate this modeling strategy for characteristic wave propagation, results are compared with 3D-NSCBC in the three 2D test cases discussed above.

\subsection{Transverse outgoing vortex}

A first validation has consisted in a configuration similar to figure 3. An outgoing vortex is convected through the right boundary, with a normal mainstream (figure 4). In this case, relaxation coefficients are respectively specified by $\widehat{\eta}_{4}=\widehat{U}_{1} / c$ and $\widetilde{\eta}_{4}=\widetilde{U}_{1} / c$ for the 3D-NSCBC or $3 \mathrm{D}-\mathrm{TOM}$ outflows. The referential $\widetilde{\mathcal{R}}$ is dynamically computed from the local velocity by

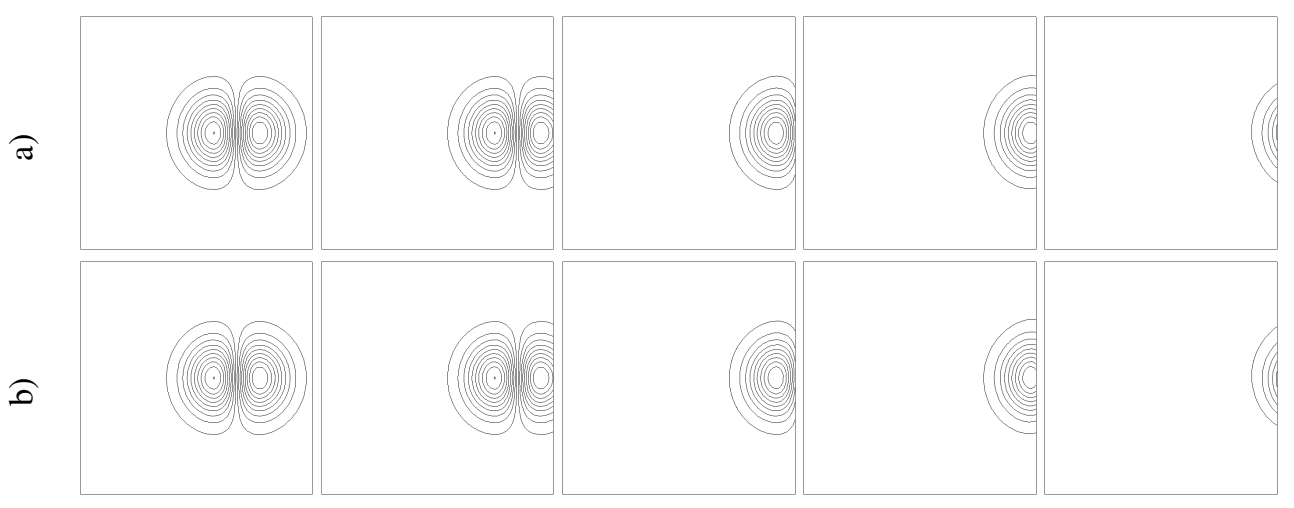

Figure 4: Outgoing vortex with mainstream normal to the right outflow $\left(\widehat{U}_{1}^{i n} / c=0.575, \widehat{U}_{2}^{i n} / c=0.575\right.$ et $\left.U_{\theta}^{m} / c=0.121\right)$. a) iso- $U_{2}, 3 \mathrm{D}-\mathrm{NSCBC}$ outflow ; b) iso- $U_{2}, 3 \mathrm{D}-\mathrm{NSCBC}-\mathrm{TOM}$ outflow. 
$\widetilde{\boldsymbol{x}}_{\mathbf{1}}=\mathbf{U} / U$. The results obtained with both methods are almost indistinguishable and 3D-NSCBC is hence the effective limit of 3D-NSCBC-TOM, when the mainstream is normal to the boundary. Note that in Lodato et al. [5], the relaxation coefficient $\widehat{\eta}_{4}=\widehat{U}_{1}^{i n} / c$ is constant along the outflow and is estimated from the input mainstream value. Since $U_{\theta}^{m}<<\widehat{U}_{1}^{i n}$ in this case, this static setup of coefficient gives nearly the same behavior (not shown here) than the used dynamic set-up. Nevertheless, this static set-up has been found to generate less disturbances on density fields and

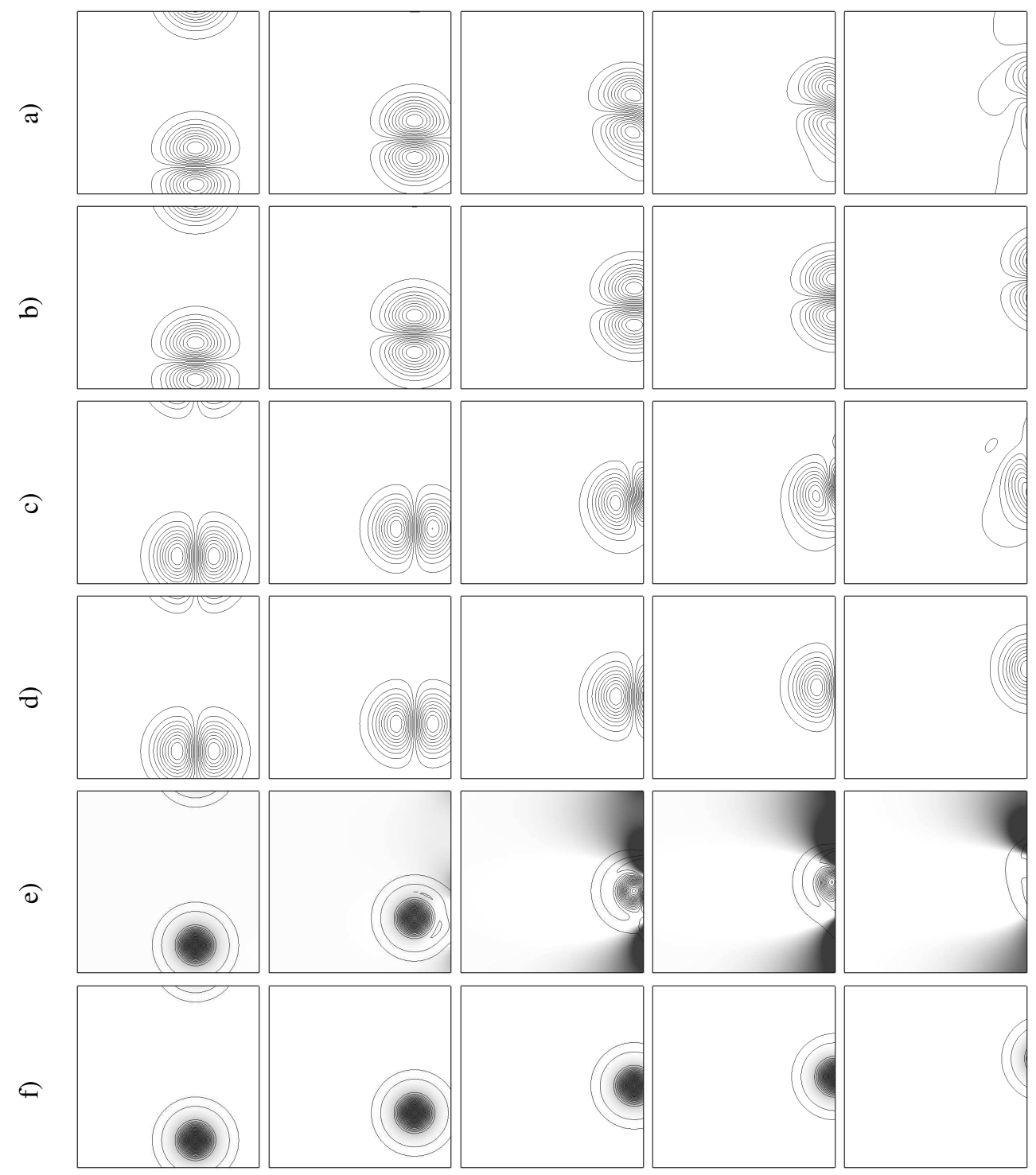

Figure 5: Outgoing oblique vortex $\left(\widehat{U}_{1}^{i n} / c=\widehat{U}_{2}^{i n} / c=0.1\right.$ and $\left.U_{\theta}^{m} / c=0.0066\right)$. a) iso- $U_{1}, 3 \mathrm{D}-\mathrm{NSCBC}$; b) iso- $U_{1}$, $3 \mathrm{D}-\mathrm{NSCBC}-\mathrm{TOM}$; c) iso- $U_{2}, 3 \mathrm{D}-\mathrm{NSCBC}$; d) iso- $U_{2}, 3 \mathrm{D}-\mathrm{NSCBC}-\mathrm{TOM}$; e) iso- $Q$ criterion and density (grey gradients), 3 D-NSCBC; f) iso- $Q$ criterion and density, 3 D-NSCBC-TOM. 
will then be used in following vortex cases. In next vortex test cases, relaxation coefficients are then prescribed by $\widehat{\eta}_{4}=\widehat{U}_{1}^{i n} / c$ and $\widetilde{\eta}_{4}=\left\|\mathbf{U}^{i n}\right\| / c$ with $\mathbf{U}^{i n}=\widehat{U}_{1}^{i n} \widehat{\boldsymbol{x}}_{1}+\widehat{U}_{2}^{i n} \widehat{\boldsymbol{x}}_{\mathbf{2}} ;$ the streamline based frame $\widetilde{\mathcal{R}}$ is statically prescribed by $\widetilde{\boldsymbol{x}}_{\mathbf{1}}=\mathbf{U}^{\text {in }} /\left\|\mathbf{U}^{i n}\right\|$.

Figure 5 shows density field, isolines of velocity components and isovalues of $Q$ criterion (i.e. the 2nd invariant of the velocity gradient tensor, see [20]), for the outgoing vortex flowing transversely through the right exit $\left(\widehat{U}_{1}^{i n}=\widehat{U}_{2}^{i n}\right)$. The $Q$ criterion is positive at vortex center (where rotational energy is concentrated), then becomes negative at the rim (where flow is sheared) and vanishes at infinity (no velocity gradient). The results of figure 5 evidence a significant improvement of the expected behavior of the non reflecting outlet: 3D-NSCBC-TOM brings (in this case) much less distortion of the presented isovalues.

A parametrical study is now conducted to investigate the behavior of 3D-NSCBC-TOM outflows on a wider range of flow conditions. A vortex test with a mainstream $U_{1}^{\text {in }} / c=0.2$, an incidence angle $\alpha=45^{\circ}$, a tangential velocity $U_{\theta}^{m} / c=0.06$ and a grid refinement $R_{V} / \Delta x=10$ is taken as a reference. The corresponding Reynolds number is $\mathrm{Re}=U_{\theta}^{m} R_{v} / v=1740$. The vortex is initially located at the center of the domain. Periodic conditions are used for horizontal boundaries while NSCBC, 3D-NSCBC and the present 3D-NSCBC-TOM treatment are used for the right boundary. Figure 6 plots the density field and some iso- $Q$ criterion when the center of the vortex reaches the outflow boundary for different incidence angles and for these different outflow treatments. It shows that NSCBC outflows introduces an important level of distortions whatever

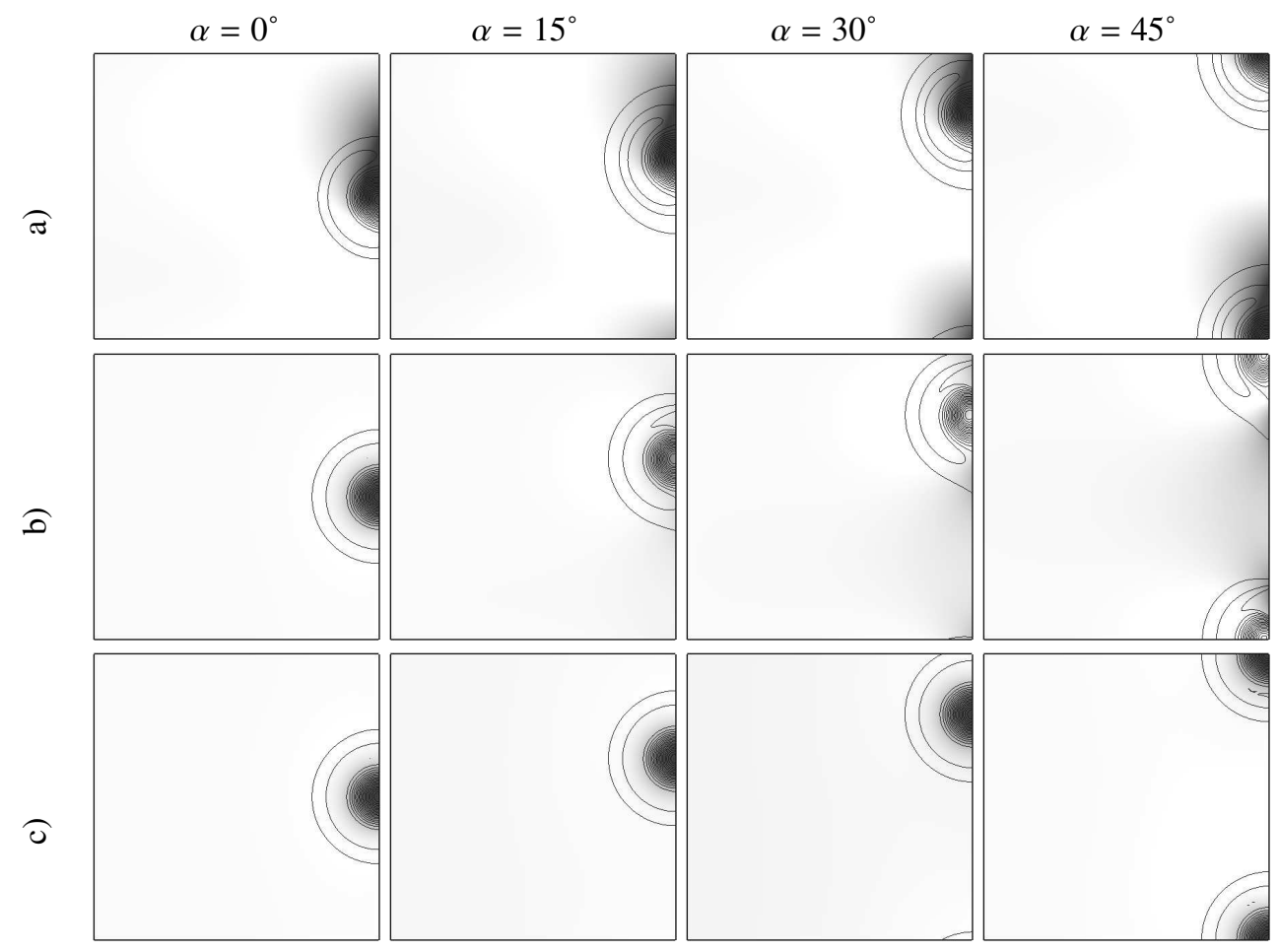

Figure 6: Outgoing oblique vortex with different incidence angles $\alpha=\operatorname{atan}\left(U_{2}^{i n} / U_{1}^{i n}\right)\left(U_{1} / c=0.2\right.$ and $\left.U_{\theta}^{m} / c=0.061\right)$. The density field (grey gradients) and iso-values of the $Q$ criterion are plotted at time $t=2.5 \cdot c /\left(10 R_{V}\right)$. a) NSCBC. b) 3D-NSCBC. c) 3D-NSCBC-TOM. 
the incidence angle ; 3D-NSCBC strongly reduces these distortions for small incidence angles but still generates strong disturbances for $\alpha>15^{\circ}$. The present 3D-NSCBC-TOM treatment drastically decreases these distortions even for high incidence angles.

The accuracy of the solution is then measured when varying one of this four parameters for NSCBC, 3D-NSCBC and the present 3D-NSCBC-TOM strategies. The maximum difference between velocity $U_{1}$ (resp. $U_{2}$ ) and its analytic solution $U_{1}^{a n a}$ (resp. $U_{2}^{a n a}$ ) defines a velocity error which represents the quality of the solution. A maximum error can similarly be defined between the simulated density $\rho$ and its analytic solution $\rho^{\text {ana }}$. Figure 7 plots the time evolution of these spatial maximum errors for $\alpha=0^{\circ}$ and $45^{\circ}$. Note that pressure field is relatively similar to density field for all these simulations. The normalized pressure error $\left(P-P^{a n a}\right) / P^{i n}$ which has been found identical to the normalized density error $\left(\rho-\rho^{a n a}\right) / \rho^{i n}$ is not plotted to facilitate the reading. These curves contain most of previous observations : NSCBC outflows generates strong errors whatever the incidence angle ; 3D-NSCBC and 3D-NSCBC-TOM are both accurate for $\alpha=0^{\circ}$; for higher incidence angles, 3D-NSCBC generates strong distortions while maximum errors of all properties are still very low with the presented 3D-NSCBC-TOM outflow.
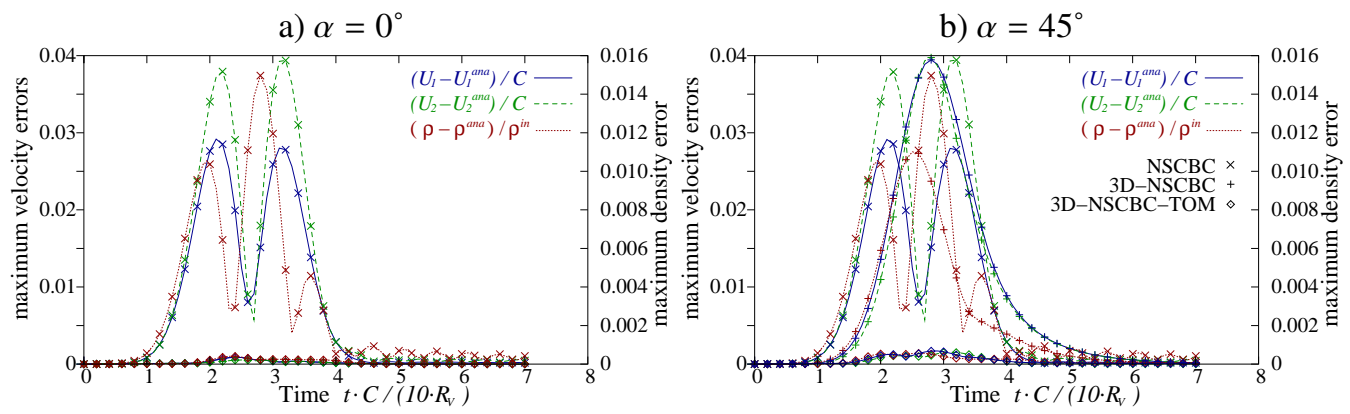

Figure 7: Evolution of some maximum errors during the exit of a vortex through a face for different outflow treatments. Errors on velocities $U_{1}, U_{2}$ and density $\rho$ are respectively plotted in blue, green dashed and red dotted lines ; the present 3D-NSCBC-TOM strategy (diamond symbols) is compared to NSCBC and 3D-NSCBC strategies (crosses). a) mainstream normal to boundary $\alpha=0$, e.g. $\widehat{U}_{2}^{i n}=0$. b) mainstream transverse to boundary $\alpha=45^{\circ}$, e.g. $\widehat{U}_{2}^{i n}=\widehat{U}_{1}^{i n}$.

These maximum errors reach maximum values when the vortex is crossing the boundary. Total maximum errors can therefore be defined as the temporal maximum of these spatial maximum errors for velocities $U_{1}, U_{2}$ and density $\rho$. We then performed 420 numerical simulations of outgoing vortices to measure these maximum errors for a wide range of incident angle $\left(\alpha \in\left[-90^{\circ} ; 90^{\circ}\right]\right)$, a wide range of tangential vortex velocities $\left(U_{\theta}^{m} / U_{1}^{i n} \in[-2 ; 2]\right)$, different grid resolutions $\left(R_{V} / \Delta x \in[2 ; 20]\right)$, different advection velocities $U_{1}^{i n} / c \in[0.01 ; 0.5]$ and for three different outflow treatments. All these errors are plotted on figure 8 .

Figure 8a that shows the influence of the incidence angle confirms that 3D-NSCBC-TOM and 3D-NSCBC outflow are similar for a normal mainstream $\left(\alpha=0^{\circ}\right)$. It also indicates that maximum errors quickly increase for transverse mainstream with 3D-NSCBC and these errors are always very high with NSCBC outflows. Errors on velocities, density and pressure are drastically decreased for transverse flows with the present strategy. Nevertheless, some oscillations appeared for too high incidence angles $\left(|\alpha| \geq 60^{\circ}\right)$ with 3D-NSCBC outflows. Figure 8b demonstrates that 3D-NSCBC-TOM reduces distortions even for strong vortices. Nevertheless, maximum errors may become important for too high tangential velocities but velocity errors are still under $3 \%$ of $U_{1}^{i n}$ for the present strategy when all $U_{1}$ velocities remain positive $\left(\left|U_{\theta}^{m} / U_{1}^{i n}\right| \leq 1\right)$. 

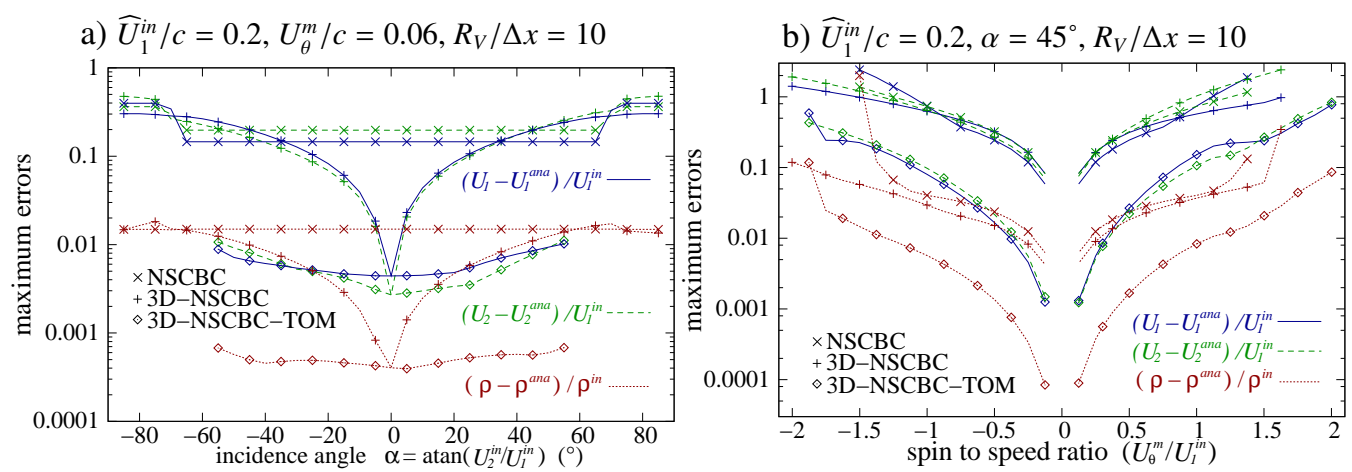

c) $\widehat{U}_{1}^{i n} / c=0.2, U_{\theta}^{m} / c=0.06, \alpha=45^{\circ}$
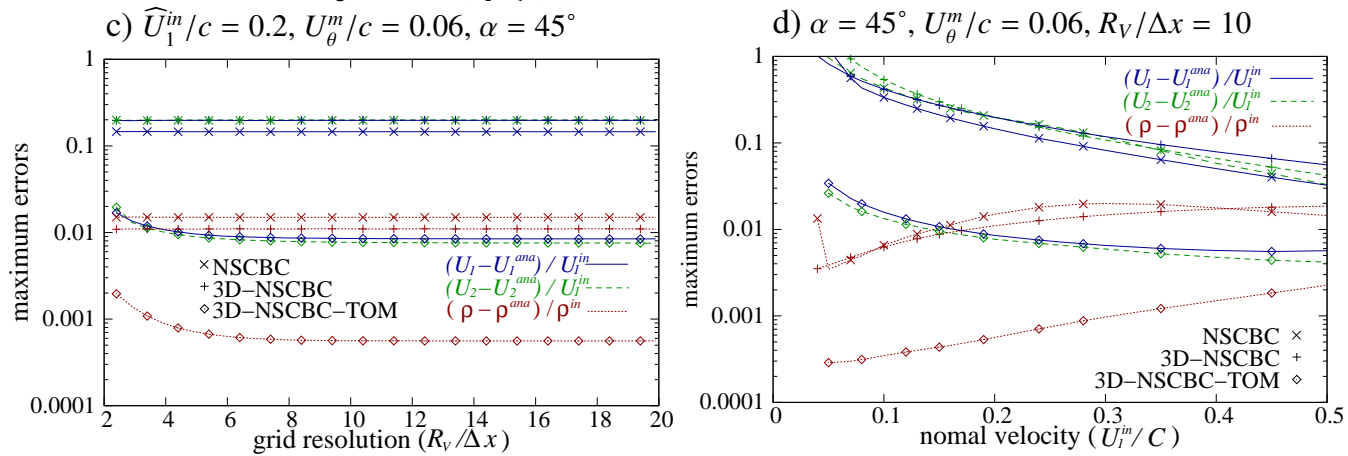

Figure 8: Parametrical study of an outgoing vortex through a face. Comparisons of maximum errors of velocities and density for different outflow treatments. Errors on velocities $U_{1}, U_{2}$ and density $\rho$ are respectively plotted in blue, green dashed and red dotted lines ; the present 3D-NSCBC-TOM strategy (diamond symbols) is compared to NSCBC and 3D-NSCBC strategies (crosses). a) influence of the incidence angle. b) influence of the spin to speed ratio. c) influence of the grid refinement. d) influence of the mainstream velocity.

For higher tangential velocities, temporary negative velocities may generate some distortions but simulations remain stable until $\left|U_{\theta}^{m} / U_{1}^{i n}\right|<2$ for 3D-NSCBC and 3D-TOM treatments. Figure $8 \mathrm{c}$ shows that grid refinement does not have a significant importance to reduce these maximum errors caused by the outflow treatment. Figure 8a demonstrates that NSCBC and 3D-NSCBC satisfyingly solves this transverse moderate vortex for high Mach numbers (typically $U_{1}^{i n} / c>0.4$ ). The present 3D-TOM strategy gives pertinent results even for low intensity mainstream. All treatments become unstable for too low mainstreams, e. g. when $U_{1}^{i n}<U_{\theta}^{m}$.

\subsection{Corner outflow}

The behavior of 3D-NSCBC is now tested for a vortex that is convected through the upright corner of the computational domain. Mainstream components are chosen as $\widehat{U}_{1}^{i n} / c=\widehat{U}_{2}^{i n} / c=$ 0.2 ; vortex intensity is such that $U_{\theta}^{m} / c=0.06$. Figure 9 shows isovalues of the $Q$-criterion when the vortex goes through the upright corner. In figure $9 \mathrm{a}$, right and up boundaries are treated with 3D-NSCBC "standard" model, figure 9b presents results with 3D-NSCBC-TOM.

As already announced at the end of subsection 4.1, figure 9a shows vortex distortion when the outflow is treated with 3D-NSCBC. In this case, the acoustic force introduced by 3D-NSCBC outflow model is not a sufficiently good approximation of the "real" one, allowing a pertinent non-reflecting outflow. The $Q$-criterion is particularly distorted in shear flow zones and after vortex outlet crossing. 


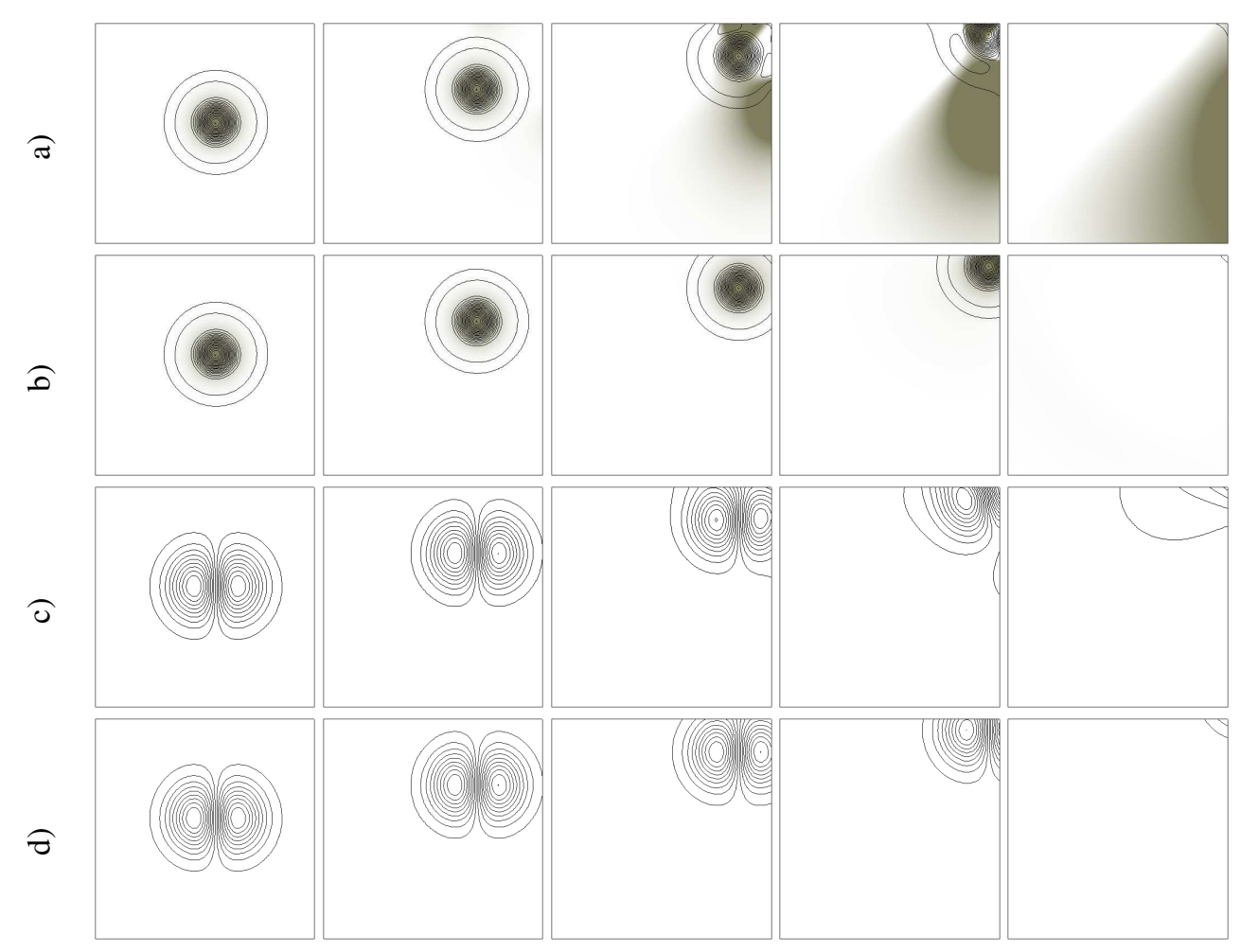

Figure 9: Outgoing vortex at upright corner $\left(U_{1} / c=0.2\right.$ and $\left.U_{\theta}^{m} / c=0.06\right)$. a) density field (grey gradients) and $Q$ criterion iso-lines, 3D-NSCBC; b) density field and $Q$-criterion iso-lines, 3D-NSCBC-TOM; c) iso- $U_{2}, 3 \mathrm{D}-\mathrm{NSCBC}$; $)$ iso- $U_{2}, 3 \mathrm{D}-\mathrm{NSCBC}-\mathrm{TOM}$.

In this case, 3D-NSCBC modeling induces quite large vortex deformations. With proposed 3D-NSCBC-TOM outflow treatment, the vortex is very moderately warped and the simulation is numerically stable (compared to 3D-NSCBC-TO).

\subsection{Expanding flame revisited}

The expanding premixed laminar flame is computed with 3D-NSCBC-TOM. Figure 10 represents density, velocity vectors, temperature and reaction rate evolution for both 3D-NSCBC and 3D-NSCBC-TOM strategies.

As already discussed in [4], quite early a square-shaped flame is obtained with 3D-NSCBC. It is worth noticing that, with present 3D-NSCBC-TOM, the flame shape remains circular. In this case, the flow acceleration seems quite pertinently modeled, even when the flow is not normal to the outlet.

\section{Concluding Remarks}

Modified 3D-NSCBC outflow treatments for compressible Navier-Stokes multi-dimensional computations, including outflow corners, have been discussed. By analyzing simple, but discriminating, 2D test cases, namely exiting vortex (with an oblique mainstream velocity with respect 

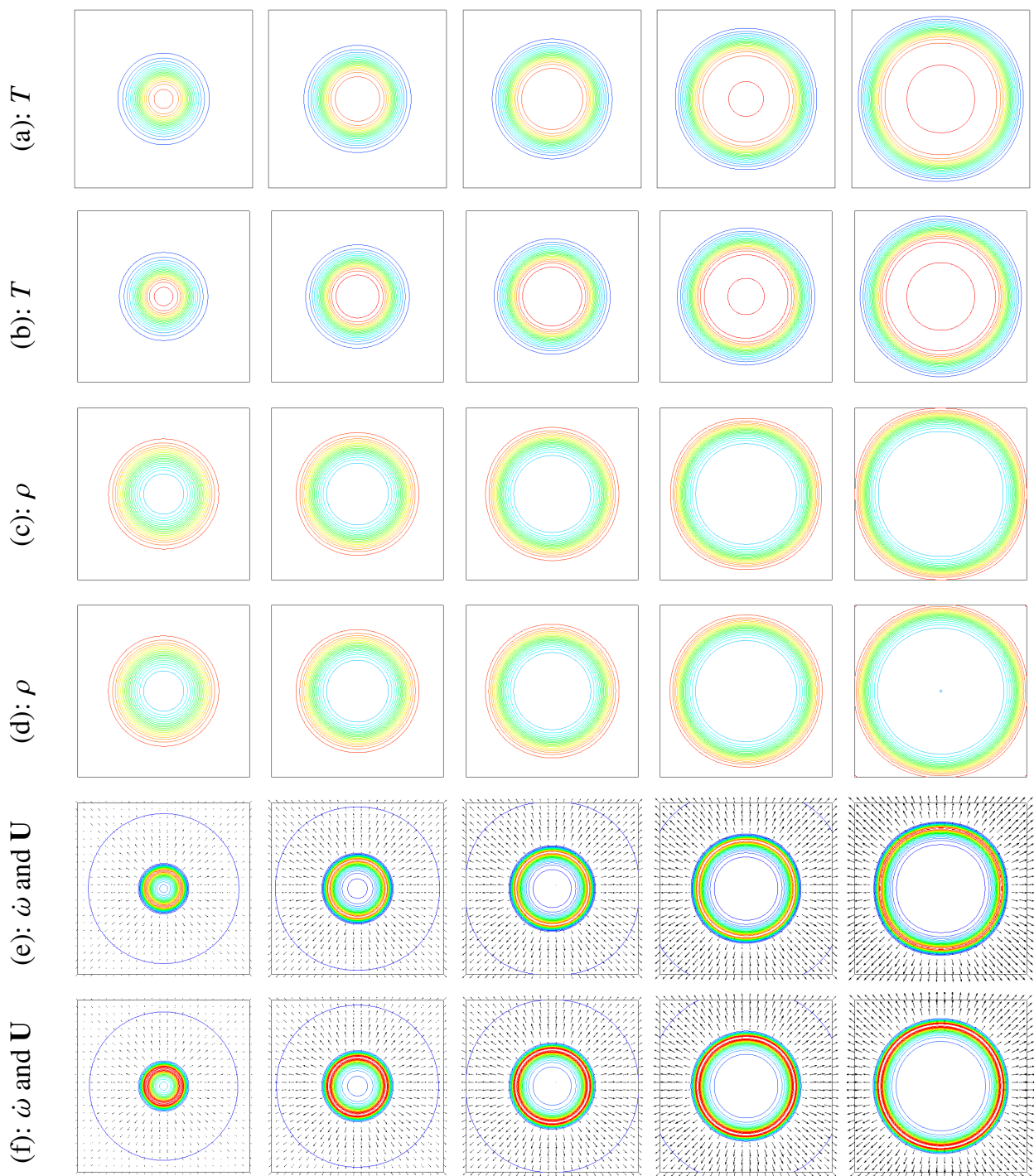

Figure 10: 2D expanding laminar premixed flame. a, c, e: 3D-NSCBC. b, d, f: 3D-NSCBC-TOM. a, b: Temperature, c, d: Density, e, f: Reaction rates and velocity vectors.

to the boundary) and expanding laminar flame, it is first shown that flow acceleration seems not correctly taken into account when the mainstream exiting the computational domain is not normal to the outlet boundary. This evidenced the importance of the choice of the chosen coordinate system when modeling characteristic wave amplitude propagation through NSCBC procedure. This outflow treatment can be improved by decomposing characteristic wave amplitude propagation in a coordinate system aligned with the mainstream line, which is not necessarily normal to the outlet boundary. It is important to note, however, that the streamlines decomposition conceptually privileges the entropy wave with respect to the acoustic waves and the targeted applications 
should be more directly related to combustion than aeroacoustics. By comparing these different models, each of them may be interpreted in terms of different prescribed external acoustic forces exerted on the fluid.

Along these lines a new strategy (3D-NSCBC-TOM) is proposed that noticeably improves non reflecting outflow behavior, including outflowing corners. Transverse outflowing vortices are much less warped at exit vicinity than with 3D-NSCBC treatment and the shape of an expanding flame is much less square-like at boundary vicinity. Another challenging perspective for 3D-NSCBC would be to design a formulation that may allow a coordinate system-independent modeling.

\section{Acknowledgements}

We thank the referees for their careful analysis of our manuscript and their valuable comments and suggestions. One of the author (EA) has been supported by a grant provided by the French ministry of Higher Education and Research.

\section{References}

[1] K. Thompson, Time-dependent boundary conditions for hyperbolic systems I, Journal of Computational Physics 68 (1987) 1-24.

[2] T. Poinsot, S. Lele, Boundary conditions for direct simulations of compressible viscous flows, Journal of computational physics(Print) 101 (1) (1992) 104-129.

[3] C. Yoo, Y. Wang, A. Trouvé, H. Im, Characteristic boundary conditions for direct simulations of turbulent counterflow flames, Combustion Theory and Modelling 9 (4) (2005) 617-646.

[4] C. S. Yoo, H. G. Im, Characteristic boundary conditions for simulations of compressible reacting flows with multidimensional, viscous and reaction effects, Combustion Theory and Modelling 11 (2) (2007) 259-286.

[5] G. Lodato, P. Domingo, L. Vervisch, Three-dimensional boundary conditions for direct and large-eddy simulation of compressible viscous flows, Journal of Computational Physics 227 (10) (2008) 5105-5143.

[6] G. Lodato, L. Vervisch, P. Domingo, A compresssible wall-adapting similarity mixed model for large-eddy simulation of the impinging round jet, Phys. Fluids 21 (2009) 035102.

[7] Q. Liu, O. Vasilyev, Nonreflecting boundary conditions based on nonlinear multidimensional characteristics, International Journal for Numerical Methods in Fluids.

[8] F. Nicoud, Defining wave amplitude in characteristic boundary conditions, J. Comput. Phys. 149 (1999) $418-422$.

[9] L. Selle, F. Nicoud, T. Poinsot, The actual impedence of non-reflecting boundary conditions: Implications for the computations of resonators, AIAA J. 42 (5) (2004) 958-964.

[10] J. Sutherland, C. Kennedy, Improved boundary conditions for viscous, reacting, compressible flows, J. Comput. Phys. 191 (2) (2003) 502-524.

[11] R. Prosser, Towards improved boundary conditions for the dns and les of turbulent subsonic flows, J. Comput. Phys. 222 (2) (2007) 469-474.

[12] J. Kim, D. Lee, Generalized characteristic boundary conditions for computational aeroacoustics, AIAA journal 38 (11) (2000) 2040-2049.

[13] C. Laney, Computational gas dynamics, Cambridge Univ Pr, 1998.

[14] C. Hirsch, Numerical computation of internal and external flows: fundamentals of computational fluid dynamics.

[15] E. Albin, Y. D’Angelo, L. Vervisch, Using staggered grids with characteristic boundary conditions when solving compressible reactive Navier-Stokes equations, International Journal for Numerical Methods in Fluids (2010) .

[16] L. Guichard, L. Vervisch, P. Domingo, Two-dimensional weak-shock vortex interaction in a mixing zone, AIAA Journal 33 (10) (1995) 2539-2544.

[17] P. Domingo, L. Vervisch, DNS of partially premixed flame propagating in a turbulent rotating flow, Proc. Combust. Inst. 31 (2007) 1657,1664.

[18] J. Sutherland, C. Kennedy, Improved boundary conditions for viscous, reacting, compressible flows, Journal of computational physics 191 (2003) 502-524.

[19] T. Colonius, S. K. Lele, P. Moin, The free compressible viscous vortex, Journal of Fluid Mechanics 230 (1991) 45-73.

[20] Y. Dubief, F. Delcayre, On coherent-vortex identification in turbulence, Journal of Turbulence 1 (1) (2000) 1-22. 